\title{
tripleC-Interview mit Kerem Schamberger über Berufsver- bote, linke Kommunikationswissenschaft und Kritik an der deutschen Wissenschaftslandschaft
}

\begin{abstract}
Kerem Schamberger ist ein Doktorand am Institut für Kommunikationswissenschaft und Medienforschung der Ludwig-Maximilians-Universität München. Er ist auch ein linker Aktivist und Mitglied der Deutschen Kommunistischen Partei (DKP). Im Zuge seiner Anstellung wurde auf Basis der bayrischen Gesetzeslage vom Bayrischen Verfassungsschutz geprüft, ob Kerem als verfassungsfeindlich gilt und inm daher die Anstellung verweigert werden sollte. In der deutschen Mediensoziologie gab es mit Horst Holzer in den 1970er-Jahren einen ähnlichen Fall eines Berufsverbots, das damals durchgesetzt wurde. tripleC hat ein Interview mit Kerem geführt und inn gebeten, die Hintergründe genauer zu erläutern.
\end{abstract}

Das Interview wurde von Christian Fuchs und Thomas Allmer auf Deutsch geführt und ins Englische übersetzt.

tripleC: Kerem, kannst du mehr über deine wissenschaftlichen Interessen sagen, welche Forschung du durchführst und die politischen Aspekte dieser Themen.

Kerem Schamberger: Mein Schwerpunkt liegt derzeit auf Mediensystemforschung, der Analyse von Medienfreiheit und Autonomie von Journalisten. Dabei ist es wichtig, sich vom rein liberalen Verständnis der Pressefreiheit zu lösen, so wie sie von vielen NGOs, aber auch teilweise in der Kommunikationswissenschaft vertreten wird. Hier werden oft der Staat und mögliche staatliche Eingriffe als primäre Bedrohung der Unabhängigkeit von Journalisten und Medien gesehen. Diese ist zwar real, allerdings geraten in vielen Medienfreiheitsindizes, wie zum Beispiel von IREX oder Freedom of the Press, andere wichtige Einschränkungen der Medienfreiheit aus dem Blick. Die implizite (markt)liberale Einstellung führt zu einem blinden Fleck in Sachen Eigentum. Medieneigentumskonzentration und die damit verbundene Einschränkung der Medienfreiheit wird nicht wahrgenommen. Die Kolleginnen Sapiezynska und Lagos (2016) haben die Medienfreiheitindizes von Freedom House und Reporter ohne Grenzen untersucht und sind zu dem Schluss gekommen, dass diese in der Analyse demokratischer Länder Lücken aufweisen, da sich die Indizes vor allem auf staatliche Beschränkungen und Angriffe auf Journalisten konzentrieren und ökonomische Einschränkungen, wie Medieneigentumsstrukturen oder Abhängigkeiten vom Anzeigenmarkt zu kurz kommen. Bezeichnenderweise kommen westliche MedienfreiheitsOrganisationen trotz unterschiedlicher Ansätze und Methoden, oft zu sehr ähnlichen Ergebnissen, die sich in der Weltkarte der Pressefreiheit von Freedom of the Press sehr symbolisch ausdrücken: Im „Westen” ist die Freiheit groß, im Rest der Welt gering. Welche negative Rolle die Konzentration von Medieneigentum dabei bei uns spielt, wird nicht thematisiert.

Letztes Jahr habe ich dazu in Uganda geforscht und bin zu dem Ergebnis gekommen, dass dort Einschränkungen der Medienfreiheit weniger durch direkte Staatseingriffe als durch die schlechten Arbeitsbedingungen und politische und wirtschaftliche Interessen der Medieneigentümer erfolgen. 
Außerdem beschäftige ich mich noch mit Diskursanalyse, also mit dem Sagbaren und Nicht-Sagbaren in unserer Gesellschaft und wie Diskurse Dinge überhaupt erst real werden lassen.

tripleC: In wie fern siehst du dich in der Tradition der kritischen Medien- und Kommunikationswissenschaft? Würdest du dich selbst als kritischer Forscher bezeichnen?

Kerem: Mein Anspruch ist es ein kritischer Kommunikationswissenschaftler zu sein. Dazu gehört vor allem auch die Marx'sche Gesellschaftsanalyse und kritische politische Ökonomie, angewendet im Bereich der Kommunikationswissenschaft. Aber noch gibt es eine Diskrepanz zwischen meinem Anspruch und der Wirklichkeit. An unserem Institut in München, wie in der deutschen Kommunikationswissenschaft generell, gibt es keinen in diesem Sinne kritischen Strang. Das Bachelorstudium lässt zeitlich keinen Raum sich neben dem offiziellen Lehrkanon noch viel mit anderen „randständigen” Theorien zu beschäftigen. Im Masterstudium ist man dann sehr davon abhängig, was der oder die Professor/in forschen will. Auch wenn das in meinem Fall viel Spaß gemacht hat, so konnte ich mich kurz gesagt in meinem bisherigen Studium noch nicht in den marxistischen Bereich der Kommunikationswissenschaft einarbeiten, den es ja zum Glück gibt, zum Beispiel in England und den USA, aber auch in Russland und Indien. In Deutschland wurden Wissenschaftler, die sich als Marxisten bezeichnen, in der Vergangenheit konsequent politisch und strukturell aus den Universitäten gedrängt. Entsprechende Nachwuchswissenschaftler/innen konnten keinen Fuß fassen.

Insgesamt kommt mir die Beschäftigung mit großen Gesellschaftstheorien, unter anderem dem Marxismus, aber nicht nur, bei uns im Fach zu kurz. Man arbeitet sich lieber an Theorien mittlerer Reichweite ab und versucht Reputation durch Anhäufung von Zahlen zu erlangen. Ich finde das reicht nicht aus, vor allem wenn wir uns die derzeitige gesamtgesellschaftliche Entwicklung anschauen. Wissenschaften, auch unsere, haben die Aufgabe Gesellschaftskritik zu üben und entsprechende Lösungsvorschläge zu entwerfen.

Ich mache es mir jetzt zur Aufgabe, mich in den kritischen Teil der Kommunikationswissenschaft nach und nach einzuarbeiten. Dabei bekomme ich zum Glück Hilfe. Zum Beispiel vom Kollegen Thomas Allmer von der University of Stirling. Gemeinsam führen wir im kommenden Semester in München ein Seminar zum Thema „Soziale Medien und Kapitalismus" durch.

tripleC: Kannst du auch über dein politisches Leben sprechen und darüber, wie du politisiert wurdest? Welche persönlichen Erfahrungen haben dich dabei geprägt?

Kerem: Ich bin politisch aktiv seit ich 15 Jahre alt war. Damals habe ich viel gelesen, auch die Klassiker Marx, Engels und Lenin. Ich glaube den Ausschlag hat damals ein Geburtstagsgeschenk meines Vaters gegeben, eine Biographie von Che Guevara. Danach dachte ich, man muss etwas gegen die Ungerechtigkeit der Welt tun. Dazu gehört nicht vereinzeltes vor sich hinarbeiten, sondern Organisierung. Also mit anderen Menschen gemeinsam für eine Sache eintreten. Damals, im Dezember 2001, bin ich als Konsequenz dann in die Sozialistische Deutsche Arbeiterjugend (SDAJ) eingetreten. Ungefähr 2003 bin ich dann in die Deutsche Kommunistische Partei (DKP). Mich hat dabei vor allem die Verbindung aus marxistischer Theorie und konkreter Praxis interessiert. Es ist ja kein Geheimnis, dass die DKP eine ziemlich überalterte 
Partei ist, aber das hat den Vorteil, dass in ihr ganz viel Kampferfahrung steckt, aus der Zeit des deutschen Nazi-Faschismus, der Adenauer-Ära und der Wiederaufrüstung und so weiter. Das beeindruckt mich heute noch. Auch wenn ich unsere eigene Geschichte und auch die derzeitige Entwicklung meiner Partei sehr kritisch sehe. Wir müssen zu den Verbrechen der kommunistischen Bewegung stehen, diese Aufarbeiten und daraus Lehren für die heutige Zeit ziehen. Ich glaube das haben noch nicht alle in der DKP verstanden.

Die Praxis lebe ich eher außerhalb der DKP. Als ich vor einem Studium eine Ausbildung zum Veranstaltungskaufmann gemacht habe, war ich im Betriebsrat. Im Studium war ich aktiv in der Fachschaft, außerdem bin ich in einigen anderen zivilgesellschaftlichen Organisationen mal mehr mal weniger aktiv. Seit einiger Zeit beschäftigt mich die schlimme Entwicklung in der Türkei sehr, auch weil die Hälfte meiner Familie aus diesem Land kommt.

tripleC: Als dir eine finanzierte Doktorandenstelle an der Ludwig-MaximiliansUniversität München (LMU) angeboten wurde, wurde ein Rechtsprozess in Gang gesetzt, der in einem Berufsverbot resultieren hätte können. Kannst du mehr über den Hintergrund sagen? Wie bist du informiert worden? Was sind deiner Ansicht nach die zu Grunde liegenden Motive? Wie sieht die Situation im Moment aus?

Kerem: Als ich mich im Juli 2016 für die Stelle als Wissenschaftlicher Mitarbeiter am Lehrbereich Meyen beworben habe und genommen wurde, musste ich einen sogenannten „Bogen zur Prüfung der Verfassungstreue” ausfüllen. Dort sind Organisationen aufgelistet, die nach Meinung des bayerischen Verfassungsschutzes verfassungsfeindlich sind. Dieser „Gesinnungs”-Bogen muss von allen Bewerbern des öffentlichen Dienstes in Bayern ausgefüllt werden. Skandalöserweise finden sich dort antifaschistische Organisationen, wie die Vereinigung der Verfolgten des Naziregimes (VVN), direkt neben Naziorganisationen wie der NPD oder militanten faschistischen Kameradschaften. Auch kapitalismuskritische, linke Organisationen, wie die DKP oder die Rote Hilfe sind dort aufgelistet. Wenn man dort nun ankreuzt irgendwo Mitglied zu sein, dann wird der Verfassungsschutz um Auskunft gebeten, was es mit der Person auf sich hat. So war das auch in meinem Fall.

Nur hat sich die Sicherheitsbehörde monatelang nicht bei der Universität mit Informationen zurückgemeldet. Ich konnte deshalb meine Stelle zum geplanten Termin am 1. Oktober 2016 nicht antreten. Ich hatte also ein de facto Berufsverbot. Berufsverbote, Anfang der 70er Jahre eingeführt vom sozialdemokratischen Bundeskanzler Brandt, führten zu entsprechenden Verfahren gegen zehntausend Kommunist/inn/en, Friedensaktivist/inn/en und andere Linke. Hunderttausende wurden auf ihre Gesinnung hin überprüft. In den meisten Bundesländern wurde diese Regelung nach 1989 abgeschafft oder nicht mehr angewendet. Nicht so in Bayern, das schon seit der Niederschlagung der Räterepublik 1919 die rechte Ordnungszelle in Deutschland ist.

Ich habe mich aufgrund der Verzögerungstaktik des Verfassungsschutzes dann dazu entschlossen, an die Öffentlichkeit zu gehen. Unterstützung hatte ich dabei von der ehemaligen Bundesjustizministerin und Anwältin Herta Däubler-Gmelin. Das mediale Echo war riesig, Zeitungen berichteten und sogar im Fernsehen lief ein längerer Beitrag. Ironischerweise hat der Verfassungsschutz also dafür gesorgt, dass ein Kommunist auf den Titelseiten der Zeitungen präsent war und dabei auch wieder eine Beschäftigung mit entsprechenden Themen stattgefunden hat ${ }^{1}$.

${ }^{1}$ Medienberichterstattung: 
Der Verfassungsschutz hat sich verkalkuliert. Also musste er reagieren und übermittelte der Universität endlich seine „Erkenntnisse” über mich. Darin schreibt er abschließend: „Sein Eintreten für die marxistische Lehre und seine Selbstbezeichnung als Kommunist lassen sich mit der freiheitlichen demokratischen Grundordnung der Bundesrepublik Deutschland eigentlich nicht vereinbaren." Es ist klar, dass die „Verfassungshüter", die auf dem rechten Auge blind sind, aber gegen alles Linke fast schon allergisch reagieren, mich nicht an der Uni sehen wollten. Die Universität stellte mir auf Grundlage der VS-Erkenntnisse fünf Fragen. Diese beantwortete ich gemeinsam mit meiner Anwältin und um Weihnachten herum erhielt ich die Antwort, dass die Zweifel an meiner „Verfassungstreue” nun behoben seien und ich zum 1. Januar 2017 die Stelle antreten könne. Das ist natürlich ein großer Erfolg, der ohne die vielen Solidaritätsbekundungen und den öffentlichen Druck nicht erreicht worden wäre. Dafür möchte ich mich bei allen bedanken!

tripleC: Kannst du mehr dazu sagen, welche Mitgliedschaften in politischen Organisationen du bei deiner Bewerbung angegeben hast? Was hätte passieren können, wenn du solche Informationen zurückgehalten hättest?

Kerem: Angekreuzt habe ich die Deutsche Kommunistische Partei (DKP), die Vereinigung der Verfolgten des Naziregimes - Bund der AntifaschistInnen (VVN), die Rote Hilfe und die Sozialistische Deutsche Arbeiterjugend (SDAJ), in der ich bis 2013 Mitglied war. In der Praxis ist es natürlich so, dass die wenigsten ihre Mitgliedschaft in solchen Organisationen angeben, da sie wissen, dass sie sonst Probleme bekommen. Es kommt dann auch zu keiner Überprüfung durch den Verfassungsschutz. Mein Name wird in München und Bayern allerdings mit der DKP verbunden. Ich bin seit 2010 einer der beiden Sprecher in München. Außerdem gab es 2014 einen größeren Skandal, als die Commerzbank das Konto meiner Mutter kündigte, weil ihr Sohn, also ich, Kommunist ist. Das ging durch die Medien und damit war mein Name mit der DKP verbunden².

tripleC: Wie haben die Universität München und ihre Organisationseinheiten (in Administration und Forschung) auf die eingeleitete Überprüfung reagiert? Bist du der Meinung, dass die Universität überhaupt Interesse an diesem Fall hat? Inwiefern hat die Universität ihre Mittel ausgeschöpft, um dich und wissenschaftliche Freiheit zu verteidigen? Fühlst du dich eher unterstützt oder eher im Stich gelassen? Weißt du über andere Personen an deiner Universität bescheid, die mit ähnlichen Problemen konfrontiert sind oder waren.

http://www.sueddeutsche.de/muenchen/trotz-einschaetzung-des-verfassungsschutzes-kommunistdarf-an-muenchner-universitaet-arbeiten-1.3308728

http://www.sueddeutsche.de/muenchen/kein-job-fuer-kommunisten-ein-hauch-von-kaltem-krieg-wehtdurch-die-Imu-1.3214909

http://www.sueddeutsche.de/muenchen/trotz-einschaetzung-des-verfassungsschutzes-kommunistdarf-an-muenchner-universitaet-arbeiten-1.3308728

http://www.eldiario.es/internacional/Kerem Schamberger-Universidad-Alemaniacomunista_0_572542875.html

http://operamundi.uol.com.br/conteudo/geral/45558/aluno+de+doutorado+e+impedido+de+dar+aulas+ em+universidade+da+alemanha+por+ser+comunista.shtml

http://sendika14.org/2016/11/alman-demokrasisi-naziler-devlete-solcular-disari-saffet-soylu

${ }^{2}$ http://www.sueddeutsche.de/muenchen/konto-bei-der-commerzbank-kuendigungsgrund-sohn-

1.1874422 
Kerem: Das Institut für Kommunikationswissenschaft und Medienforschung (IfKW), an dem ich angestellt werden sollte, stand immer hinter mir. Besonders Professor Michael Meyen. Sie kannten mich ja seit meiner Bachelorstudiumszeit. Meine politische Haltung habe ich nie verheimlicht, aber ich gehe damit auch nicht hausieren. Das muss jeder für sich selbst entscheiden. Die Haltung der Universitätsleitung kann ich nicht genau einschätzen. Sie sind bestimmt froh, dass das Thema jetzt vom Tisch ist. Aber es ist natürlich auch mutig, sich gegen die Empfehlung einer Sicherheitsbehörde zu stellen. Der sogenannte Verfassungsschutz hat seinen Einsatz immer gegen linke und fortschrittliche Gedanken gerichtet. Auf dem rechten Augen ist er traditionell blind.

Berufsverbote für Linke sind insgesamt selten geworden, aber als Damoklesschwert zur Kontrolle kritischen Gedankenguts sind sie immer noch wirksam. Deshalb werden die Berufsverbote auch nicht vom bayerischen Staat abgeschafft. Unruhige Zeiten stehen uns bevor. Und da ist ein solches Instrument aus Sicht der Herrschenden immer ganz nützlich.

tripleC: Die meisten unserer Leser werden nicht vertraut sein mit der Geschichte von Berufsverboten in Deutschland. Kannst du über deren Entstehung und Geschichte erzählen. Wie viele Leute waren davon in Deutschland und Bayern betroffen. Wissen wir, wie viele Wissenschaftler/innen davon betroffen waren? Wurde das Berufsverbot nicht vor einiger Zeit bereits abgeschafft? Wie stellt sich die Situation heute dar? Wie sieht die Rechtssituation in Bayern dazu konkret aus? Ist es in anderen Bundesländern unterschiedlich? Wenn ja, warum? Wenn du beispielsweise eine Doktoratsstelle in Berlin angeboten bekommen hättest, hätte es zur selben Situation kommen können?

Kerem: Insgesamt gab es etwa 3,5 Millionen Überprüfungen von Bewerbern des öffentlichen Dienstes, es kam zu 11.000 offiziellen Berufsverbotsverfahren. 1250 Bewerbungen im öffentlichen Dienst wurden deshalb abgelehnt. 265 Menschen wurden entlassen. Die große Mehrheit davon in den 70er und 80er Jahren. Wie gesagt, seit 1989 findet diese Praxis immer weniger Anwendung. Aber es gibt Ausnahmen. 2004 hat ein antifaschistisch aktiver Lehrer Berufsverbot in Baden-Württemberg bekommen. Er musste sich durch alle Instanzen klagen, bis er 2007 Recht bekam. In Bayern und Sachsen gibt es immer noch diese Bögen zur Prüfung der Verfassungstreue. Hätte ich allerdings in Berlin eine Stelle an der Universität antreten wollen, so hätte meine DKP-Mitgliedschaft dort vermutlich zu keiner Diskussion geführt. Obwohl wir dort seit Neuestem wieder einen Fall haben, als der Wissenschaftler Andrej Holm wegen angeblicher falscher Angaben zu seiner Stasi-Zugehörigkeit vor 1989, von seiner Universität entlassen wurde. Und das obwohl er einer der besten Wissenschaftler im Bezug auf kapitalistische Stadtentwicklung ist und den GentrifizierungsDiskurs maßgeblich mitgeprägt hat. Jetzt gilt es Solidarität mit inm zu zeigen.

tripleC: Es existiert eine Liste von Organisationen, die der Bayrische Verfassungsschutz als verfassungswidrig einstuft. Darin enthalten sind die Deutsche Kommunistische Partei DKP und die Studentenorganisation der Deutschen Linkspartei (Die Linke.SDS). Liegen wir richtig in der Annahme, dass auch Die Linke als Gesamtes bis zu einem gewissen Zeitpunkt auf dieser Liste zu finden war? Praktisch gesprochen bedeutet das, dass ein Mitglied dieser Organisationen keine Anstellung im öffentlichen Dienst in Bayern finden kann. Was ist der dahinterliegende Grund, solche 
Organisationen auf diese Liste stellen? Demokratischer Sozialismus ist eine ziemlich populäre Idee heutzutage, wie zum Beispiel der verhältnismäßige Erfolg von Bernie Sanders in den USA und Jeremy Corbyn in Großbritannien zeigen. Unterscheiden sich deiner Meinung nach die Positionen von der DKP und Die Linke.SDS substanziell von Sanders oder Corbyn oder stehen sie für ein vergleichbares Konzept des demokratischen Sozialismus?

Kerem: Die Partei Die Linke wird seit Mai 2016 nicht mehr auf der Liste der angeblichen Verfassungsfeinde Bayerns genannt. Das war wirklich überfällig. Aber Organisationen, die der Partei Die Linke nahestehen, zum Beispiel der Studierendenverband SDS oder auch die Linksjugend, werden nach wie vor angeführt. Das ist skandalös. Erst kürzlich hat mich ein Mitglied dieser Organisationen kontaktiert, weil es auch hier Probleme mit der Anstellung im öffentlichen Dienst gibt. Da bleiben wir dann dran. Mittlerweile hat sich ein bayernweites Bündnis gegen Gesinnungsschnüffelei gegründet, dort sind vor allem Gewerkschaften aktiv. Es setzt sich für die Abschaffung des Verfassungstreuebogens im öffentlichen Dienst ein.

Natürlich haben gesellschaftliche Bewegungen, die sich um Bernie Sanders und Jeremy Corbyn herum gebildet haben, aber auch Aktivisten von DKP und Die Linke.SDS viele Gemeinsamkeiten. Sie eint die Kritik am bestehenden Kapitalismus und die dringende Notwendigkeit des Aufbaus einer anderen Gesellschaftsordnung. Einige wollen den Kapitalismus nur reformieren und menschlicher gestalten, andere sind der Meinung, so auch ich, dass der Kapitalismus an sich nicht human gestaltbar ist. Er muss abgelöst werden durch ein solidarisches Modell des Wirtschaftens und Zusammenlebens. Aber auch wenn uns manche Dinge trennen, ist es wichtiger, nach Gemeinsamkeiten zu suchen. Denn sich zu spalten und zu streiten kann sich die gesellschaftliche Linke in Deutschland, USA oder England nicht länger leisten. Konkrete Projekte im hier und jetzt, denn so fängt es immer an, können wir schon jetzt anpacken und umsetzen.

tripleC: Der deutsche Medien- und Kommunikationssoziologe Horst Holzer war einer der wichtigsten Vertreter einer entstehenden deutschen Schule der kritischen Politischen Ökonomie von Medien und Kommunikation. Er war ein Mitglied der DKP. 1971 war er mit einem Berufsverbot konfrontiert, welches darin resultierte, inn für mehrere Professuren abzulehnen und die Verbeamtung auf Lebenszeit an der Universität München 1974 zu verwehren. Wie schätzt du den Fall Horst Holzer ein? Welche Parallelen können wir zwischen deinem und Holzers Fall ziehen? Was sagt es uns, dass 45 Jahren nach Holzers Verbot kritische Wissenschafter scheinbar noch immer mit denselben Problemen konfrontiert sind? Inwieweit glaubst du, sind die darunterliegenden Gründe für solche Verbote heute ähnlich/unterschiedlich zu jenen in den 1970ern?

Kerem: Das Berufsverbot des Genossen Horst Holzer war natürlich um einiges krasser. Er war ein exzellenter Wissenschaftler und Theoretiker. Ich bin nur ein Juniorwissenschaftler, der gerade mit seiner Doktorarbeit anfängt. Holzer den Ruf auf eine Professur zu verwehren war und ist ein Skandal. Es wird deutlich, dass marxistische Wissenschaft ab einem gewissen Zeitpunkt in Deutschland gezielt aus den Universitäten gedrängt worden ist.

Für detailliertere Informationen kann ich nur den Beitrag von Scheu und Wiedemann (2008) zur Ablehnung linker Theorien in der deutschen Kommunikationswissenschaft am Beispiel Horst Holzers empfehlen. 
tripleC: Die meisten unserer internationalen Leser werden nur bedingt über die politische Lage in Bayern und München Bescheid wissen. Manche erinnern sich vielleicht an die Münchner Räterepublik von 1919, dass die NSDAP 1920 in München gegründet wurde, und Bayern der Ort war, wo Hitlers Vorherrschaft seinen Anfang nahm. Kannst du von der heutigen politischen Situation in Bayern erzählen?

Kerem: Die CSU hat die absolute Mehrheit im bayerischen Parlament. Und sie versteht sich und das von inr regierte Bayern nach wie vor als Ordnungszelle Deutschlands. Sie hat 2016 ein sogenanntes Integrationsgesetz beschlossen, wonach alle Zuwanderer auf „die Werte und Traditionen des gemeinsamen christlichen Abendlandes“3, auf die sogenannte „Leitkultur" eingeschworen werden sollen. Ganz Bayern sei geformt von "gewachsenem Brauchtum, von Sitten und Traditionen“4. Mit Stolz verweisen CSU-Propagandisten auf die Schützenverbände aus den Bergen, die mitgeholfen hatten, die erwähnte Räterepublik zusammenzuschießen. Nun ist die Beschwörung des Christentums als angeblich zentraler Bestandteil einer deutschen Kultur wieder das Grundgesetz, die deutsche Verfassung. Die CSU bildet heute mit ihrer Propaganda eine Brücke von der Union von CDU und CSU hin zur Alternative für Deutschland (AfD) und der Pegida. Man will den sogenannten „Rechtspopulisten“ das Wasser abgraben, indem man ihre Thesen übernimmt und verstärkt. Die bayerische Regierungspartei CSU rückt die politische Elite insgesamt nach rechts.

tripleC: Es ist einerseits sehr positiv, dass dein Anstellungsvertrag mit der Universität München vor Weihnachten zu Stande gekommen ist. Andererseits war es aber kein Weihnachtsgeschenk, sondern eigentlich nur ein legitimes Recht, das dir nunmehr nach einer Öffentlichkeitskampagne zugestanden wurde. Wie beurteilst du das Ergebnis und die Perspektiven, die sich in Zukunft in Bezug auf Berufsverbote für Linke in Bayern ergeben? Könnten sich solche Fälle wiederholen?

Kerem: Natürlich ist die Anstellung an der Universität München ein Erfolg. Es ist kein Geschenk, dass ich diese Stelle antreten kann, sondern mein Recht. Beziehungsweise ist es das gute Recht meines Instituts zu entscheiden, wen es anstellt und wen nicht. Da hat der Verfassungsschutz nichts mitzureden.

Ich glaube, dass die Verzögerung der Anstellung auch deshalb so lange gedauert hat, weil sie sich genau überlegen mussten: Wenn sie mich anstellen, schaffen sie einen Präzedenzfall für alle universitären Einrichtungen in Bayern. Meine politischen Aktivitäten sind in München bekannt, viele Menschen verfolgen, was wir als Marxisten und Kommunisten organisieren und auch unsere Veranstaltungen sind in den letzten Monaten sehr gut besucht. In Zukunft wird es dem Verfassungsschutz noch schwerer fallen, bei anderen fortschrittlichen Menschen solche Verzögerungstaktiken durchzuführen oder gar ein Berufsverbot zu forcieren. Denn erstens weiß er jetzt, dass dies zu einem öffentlichen Aufschrei führen würde, der seinem sowieso schon ramponierten Ruf schadet und zweitens kann nun immer auf meinen Fall verwiesen werden. Wenn ein aktives Mitglied der DKP eine Doktorandenstelle antreten kann, wieso sollte das nicht auch ein Aktivist der Studierendenorganisation SDS (Sozialistisch-demokratischer Studierendenverband) oder ein Antifaschist, der in der VVN-

http://www.vdj.de/uploads/media/160223_BaylntG_FassungMinisterrat.pdf Ebd. 
BdA (Vereinigung der Verfolgten des Naziregimes - Bund der AntifaschistInnen) organisiert ist, tun können.

Allerdings wurde ich mittlerweile schon von einem anderen linken Aktivisten kontaktiert, bei dem es auch um eine Anstellung im öffentlichen Dienst geht und diese Person auch Probleme mit dem Verfassungsschutz zu bekommen scheint. Dies zeigt, dass es mit meiner Anstellung nicht getan ist. Der sog. Bogen über die „Pflicht zur Verfassungstreue" muss abgeschafft werden. Diesen politischen Kampf gilt es nun weiter zu führen.

tripleC: Gehen wir recht in der Annahme, dass der Verfassungsschutz in seinem Gutachten empfohlen hat, dich nicht anzustellen, die Universität dich aber trotzdem angestellt hat? Wie sehen denn dazu die Rechtsgrundlagen aus? Und wissen wir, wie der Verfassungsschutz seine Argumentation begründet hat?

Kerem: Der Verfassungsschutz schreibt in seiner Erkenntnismitteilung, und das muss man sich auf der Zunge zergehen lassen, dass er mein Eintreten für die marxistische Lehre und meine Selbstbezeichnung als Kommunist nicht mit der freiheitlich demokratischen Grundordnung der Bundesrepublik Deutschland vereinbar sieht. Der Wink mit dem Zaunpfahl ist hier eindeutig: „Stellt diesen Verfassungsfeind nicht an!“. Eine Begründung, warum der Marxismus verfassungsfeindlich sein soll, bleibt er schuldig. Ich lasse derzeit über meine Anwältin klären, ob ich die VerfassungsschutzErkenntnisse komplett veröffentlichen kann. Zu verbergen habe ich nichts und es wird deutlich, mit welchen angeblichen Beweismitteln hier eine deutsche Sicherheitsbehörde versucht, gesellschaftskritische Menschen zu stigmatisieren.

Die Universität hat sich in einer mutigen Entscheidung über die Empfehlung des Verfassungsschutzes hinweggesetzt. Davor habe ich, vor allem in den heutigen Zeiten der Rechtsentwicklung, großen Respekt. Sie kann das rein rechtlich ganz einfach tun, da ihr immer nur „empfohlen“ wird, sich an die Erkenntnisse des Verfassungsschutzes zu halten. Der komplette Bogen zur Prüfung der Verfassungstreue muss Bewerbern an der Universität nicht zwingend vorgelegt werden. Deshalb sollte es jetzt eine Diskussion darüber geben, warum diese Praxis nicht einfach geändert wird und damit diese Verunsicherung für Bewerber aufgehoben wird. Auch wenn der Bogen natürlich im ganzen öffentlichen Dienst Bayerns fallen sollte und nicht nur an den Universitäten.

tripleC: Von Berufsverboten betroffene Linke machen diesen Umstand oft nicht öffentlich, da sie weitere Repression befürchten. Dein Fall hat eine öffentliche Debatte ausgelöst. In Bayern hat sich dadurch jetzt ein Bündnis gegen Gesinnungsschnüffelei gebildet. Kannst du darüber mehr sagen?

Kerem: Ich habe vorhin schon gesagt, dass es nun gilt, den politischen Kampf gegen das Damoklesschwert Verfassungstreuebogen zu führen. Dafür hat sich das Bündnis gegründet. Es besteht aus Gewerkschaftsgliederungen, wie der Gewerkschaft für Erziehung und Wissenschaft München oder dem Fachbereich 5 von Ver.di München. Aber auch die Rote Hilfe oder die DKP München sind dabei, die ja selbst im Visier des Verfassungsschutzes stehen.

In seiner Gründungserklärung schreibt das Bündnis: „Die Politik der Berufsverbote, in deren Tradition die ,Extremismusliste' steht, richtet sich gegen gesellschaftliche Alternativen zum herrschenden Wirtschafts- und Gesellschaftssystem und versucht, diese zu kriminalisieren. Die durch die Abfrage geschaffene Atmosphäre der Gesin- 
nungsschnüffelei lässt den Eindruck entstehen, dass oppositionelle Demokrat/inn/en zu bayerischen Staatsfeind/inn/en vorverurteilt werden. Dies befördert ein Klima der Einschüchterung und Verunsicherung, welches noch durch die exemplarische Disziplinierung und Bedrohung Einzelner verstärkt wird und somit breite Kreise der Auszubildenden, der Studierendenschaft sowie des Lehrpersonals an Schulen und Hochschulen abschreckt, sich für fortschrittliche Politik einzusetzen und ihre Interessen organisiert zu vertreten."

Das ist eine sehr richtige Einstellung. Für das Bündnis gilt es jetzt, die öffentliche und mediale Diskussion zum Thema Berufsverbote zu nutzen und die Institutionen darin zu unterstützen, den Gesinnungscheck abzuschaffen.

tripleC: Vielen Dank für das Interview. Alles Gute für deine Doktoratsforschung. Halte uns über deine Forschung am Laufenden.

\section{Literatur}

Scheu, Andreas and Thomas Wiedemann, 2008. Kommunikationswissenschaft als Gesellschaftskritik. Die Ablehnung linker Theorien in der deutschen Kommunikationswissenschaft am Beispiel Horst Holzer. Medien \& Zeit 23 (4): 9-17.

Sapiezynska, Ewand and Claudio Lagos. 2016. Media Freedom Indexes in Democracies: A Critical Perspective Through the Cases of Poland and Chile. International Journal of Communication 10 549-570.

\section{Kurzbiographie Kerem Schamberger:}

Duale Ausbildung zum Veranstaltungskaufmann (IHK)/Eventmanager (2008-2011). Danach Bachelorstudium der Kommunikationswissenschaft (2011-2014). Abschlussarbeit zum KubaDiskurs in deutschen Medien. Journalistische Erfahrung durch Praktikum bei der Tageszeitung Junge Welt. Anschließend Masterstudium der Kommunikationswissenschaft (20142016) mit einer Abschlussarbeit zur Medienfreiheit zu Wahlzeiten in Uganda. Während der Studienzeit durchgehend Stipendiat der Hans-Böckler-Stiftung und aktiv in der Fachschaft Kommunikationswissenschaft. Seit 2013 ist er Mitarbeiter des Instituts für sozial-ökologische Wirtschaftsforschung (isw e.V.). Seit Januar 2017 wissenschaftlicher Mitarbeiter am Lehrbereich von Prof. Dr. Michael Meyen an der Universität München. Geplante Dissertation zum kurdischen Mediensystem.

\footnotetext{
${ }^{5}$ http://www.kerem-schamberger.de/2016/12/03/buendnis-gegen-gesinnungsschnueffelei-inmuenchen-gegruendet
} 